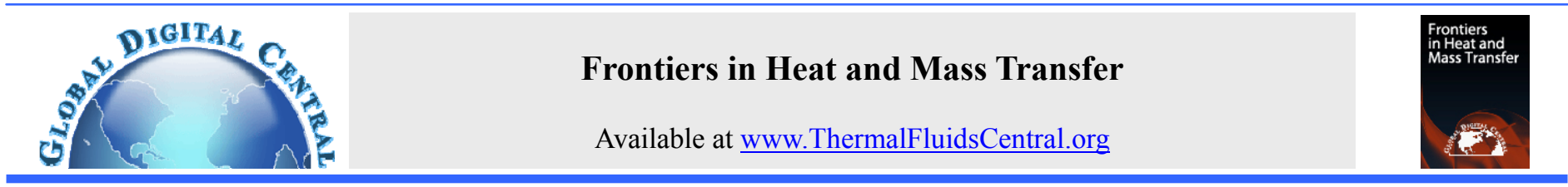

\title{
GENERAL HEAT CONDUCTION EQUATIONS BASED ON THE THERMOMASS THEORY
}

\author{
Moran Wang ${ }^{\mathrm{a}}$, Bin-Yang Cao ${ }^{\mathrm{b}}$, Zeng-Yuan Guo ${ }^{\mathrm{b}, *}$ \\ ${ }^{a}$ Los Alamos National Laboratory, Los Alamos, New Mexico 87545, USA \\ ${ }^{b}$ Department of Engineering Mechanics, Tsinghua University, Beijing 100084, China
}

\begin{abstract}
The thermomass theory regards heat owning mass-energy duality, exhibiting energy-like features in conversion and mass-like features in transfer processes. The equivalent mass of thermal energy is determined by the mass-energy equivalence of Einstein, which therefore leads to the inertia of heat in transfer. In this work, we build up a thermomass gas model based on this theory to describe the fluid-flow-like heat conduction process in a medium. The equation of state and the governing equations for transport for the thermomass gas have been derived based on methodologies of the classical mechanics since the drift speed of thermomass gas is generally far lower than the speed of light. We therefore present the general heat conduction law to describe the relationship between the heat flux and the temperature fields. The general law provides us a new viewpoint to understand the previous laws for heat conduction, such as the Fourier's law and the CV (Cattaneo-Vernotte) model. The general law will degenerate to the Fourier's law when all the thermal inertial effects are negligible or to the CV model for the unsteady heat conduction when the spacedependent inertial effects are negligible. The non-Fourier conductions, both the ultrafast heating/cooling and the ultrahigh-rate steady-state ones, have been studied using the general heat conduction law with the thermal inertial effects fully considered, and compared with the previous theoretical models and experimental data.
\end{abstract}

Keywords: Heat conduction; Thermomass theory; Thermomass gas model; Fourier's law

\section{INTRODUCTION}

In physics and thermodynamics, heat is generally known as the process of energy transfer. Heat conduction, as the most significant means of heat transfer in media, has been studied for over two hundred years. As early as 1822, Joseph Fourier, a French mathematical and physical scientist, pointed out in his famous monograph "Analytical Theory of Heat" that "these (the effects of heat) make up a special order of phenomena, which cannot be explained by the principles of motion and equilibrium", and "this part of natural philosophy cannot be connected with dynamical theories, it has principles peculiar to itself" (Fourier, 1955). He proposed the famous Fourier's law of heat conduction, stating that the time rate of heat transfer through a material is proportional to the negative gradient of temperature and to the area at right angles, through which the heat is flowing. The differential form of the Fourier's Law of heat conduction is

$$
\mathbf{q}=-k \nabla T
$$

where $\mathbf{q}$ is the local heat flux, $k$ is the thermal conductivity, and $T$ is the local temperature. The Fourier's law is very simple in mathematics and has been widely used in engineering applications and in most scientific research at life scale, even though it is only an empirical relationship.

\footnotetext{
${ }^{*}$ Corresponding author.

E-mail:demgzy@tsinghua.edu.cn(ZG);mwang@lanl.gov(MW)
}

However, when the temporal or spatial scale goes down far from normal, the applicability of the Fourier's law may be problematic. For example, for the transient heat conduction process the Fourier's law leads to an unphysical infinite heat propagation speed within the continuum field, because of its parabolic characteristics in mathematical form, which is clearly in contradiction with the theory of relativity. To overcome this contradiction of the Fourier's law, Cattaneo (Cattaneo, 1958) as a pioneer, Vernotte (Vernotte, 1958), and subsequently several others (Chester, 1963; Guyer and Krumhans.Ja, 1966; Maurer, 1969) developed a new heat conduction model, which is termed as CV (Cattaneo-Vernotte) model, to modify the Fourier's law

$\mathbf{q}+\tau_{\mathrm{CV}} \frac{\partial \mathbf{q}}{\partial t}=-k \nabla T$

where $\tau_{\mathrm{CV}}$ denotes the relaxation time and $t$ is time. This model switches the Fourier's equation from a parabolic equation to a hyperbolic one for the transient heat conduction process. The additional term in the $\mathrm{CV}$ model includes a derivative of the heat flux with respect to time, making the heat propagation speed finite. Hence, the wave nature of heat is implied, and new phenomena such as thermal resonance (Tzou, 1991; Xu and Wang, 2002) and thermal shock waves (Tzou, 1989) have been therefore found and studied. Several researchers have proved in both theory and experiment that the heat pulse propagates in continuum media as waves with a finite 
speed (Brorson, Fujimoto et al., 1987; Qiu and Tien, 1994; Tzou, 1995). Later, Gurtin and Pipkin (Gurtin and Pipkin, 1968) and Coleman et al. (Coleman, Fabrizio et al., 1982) deduced more complicated heat conduction equations similar to the $\mathrm{CV}$ model. However, there are two significant drawbacks in the CV-type models. First, such models are ad hoc mathematical approximations rather than physics-based models. The physical reality or significance of the induced parameters are not clear (Joseph and Preziosi, 1989). Second, the hyperbolic equation can violate the second law of thermodynamics. For example, consider an infinitely long wire conductor, with a heat source at the origin, and measure temperature at distances significantly remote from origin. If the heat source at origin varies with a frequency much higher than the relaxation time (i.e. faster than the speed of second sound) then the hyperbolic equation admits a temperature field in which heat would appear to be moving from cold to hot, in violation of the second law. This contradiction has been demonstrated in more mathematically rigorous forms in (Rubin, 1992; Bai and Lavine, 1995; Korner and Bergmann, 1998; Ali, 1999; Ali and Zhang, 2005). To solve the ultrafast heating on metallic films, Qiu and Tien (Qiu and Tien, 1993; Qiu and Tien, 1994) derived a phenomenological hyperbolic twostep (HTS) model when the heating duration is comparable to the electron-phonon thermal relaxation time. Tzou et al. (Tzou, 1995; Tzou, 1995; Tzou and Zhang, 1995; Tzou and Chiu, 2001) developed alternatively a dual-phase-lag model (DPL) to describe the thermal relaxation and thermalization behaviors in the ultrafast heating process in the electron gas.

Meanwhile, the Fourier's law often meets challenges at nanoscale where the microscale energy transport plays a very important role for material design (Hochbaum, Chen et al., 2008; Vining, 2008) or novel actuators (Barreiro, Rurali et al., 2008). Several theoretical and experimental studies have reported nanoscale heat transfer behavior deviating significantly from those at the normal scale (Fujii, Zhang et al., 2005; Mingo and Broido, 2005; Chen, Hochbaum et al., 2008; Donadio and Galli, 2009). Two distinguished mechanisms dominate such deviations respectively. One is caused by the atomistic effect, where the length scale in the direction of heat transfer is comparable to the molecular mean free path. In this case, the continuum assumption is not valid anymore and the energy transport has to be described by molecular dynamics or even quantum dynamics. The other mechanism lies in the high rate of heat flux resulted from extremely high temperature gradient and super low cross-section area even though the continuum assumption is still valid. The latter one is of great interests because of much more potential technical and engineering applications, but still lack of fundamental understanding.

This article is aiming to provide a common strategy to derive the general heat conduction law based on the thermomass theory (Guo, 2006; Zhang, Cao et al., 2006; Guo and Zhu, 2007). By presenting the heat conduction equation under the linear resistance assumption, we analyze the transient heat conduction process and the apparent thermal conductivity of one-dimensional nanomaterials which is a non-Fourier steady heat conduction process, and compare the results with the previous theoretical and experimental data.

\section{THERMOMASS GAS MODEL}

Heat has been generally regarded as a process of energy transfer, instead of substance transport, since the famous Caloric-Dynamic argument in the $19^{\text {th }}$ century(Mendoza, 1961). However, theoretical and experimental research since the early of the $20^{\text {th }}$ century (Nernst, 1918; Onsager, 1931; Tzou, 1995) has shown that heat owns "inertia". Tolman (Tolman, 1930) first found that heat has a weight based on the Einstein's relativity theory, which was consequently supported by other researchers (Eckart, 1940; Landau and Lifshitz, 1959). Recently Guo (Guo, 2006) has proposed a thermomass (also termed as thermal mass) theory. Based on the thermomass theory, heat has the mass-energy duality, exhibiting energy-like features in conversion processes and mass-like characteristics in transfer processes. The mass of heat is determined by the mass-energy equivalence of Einstein (Einstein, Lorentz et al., 1952), which therefore leads to the "inertia" and "weight" of heat in heat transfer. Because the mass of heat is extremely small $\left(10^{-16} \mathrm{~kg}\right.$ for $1 \mathrm{~J}$ heat $)$, it has been seldom measured but may show its significance in ultrafast heating or ultrahigh-rate heat transfer processes. Distinguished from the traditional Caloric theory, the thermomass theory treats heat as a flux of thermomass.

In this work, we develop a thermomass gas model based on the thermomass theory. We assume that the heat transfer process can be treated as a thermomass gas flow in medium, driven by a thermomass pressure gradient (a potential field). The thermomass gas is a gas-like collection of massive thermons, which is defined as a unit quasiparticle carrying the thermal energy. The thermons are supposed to be attached on the fundamental particles (molecules or atoms) of the medium for gases and liquids. For solids, the thermomass gas will be the phonon gas for crystals, attached on the electron gas for pure metals, or just between both for most of other solids. To concern the heat transfer behavior in medium, we only focus on the macroscopic flow characteristics of the thermomass gas rather than the details of each single thermon, and therefore we suppose the thermomass gas as a continuum and its transport process can be described by the classical Newton's mechanics.

\subsection{Equation of State}

Similar as the real gas, the equation of state (EOS) is complicated for the thermomass gas, especially for the liquids. A general form of the EOS of the thermomass gas can be simply written as a function of

$F\left(p_{\mathrm{T}}, \rho_{\mathrm{T}}, T, \xi\right)=0$

where $p_{\mathrm{T}}$ is the thermomass pressure, $\rho_{\mathrm{T}}$ the density of thermomass gas, $T$ the local temperature, and $\xi$ denotes the effects of interaction between thermons. When the interaction between thermons is negligible, the EOS may have an explicit form. Guo has derived the EOS for the thermomass gas in the ideal gas (Guo, 2006)

$p_{\mathrm{T}}=\kappa \rho_{\mathrm{T}} C T$

where $\kappa$ denotes the ratio of specific heats and $C$ is the specific heat capacity. Guo et al. (Guo, Cao et al., 2007; Guo and Hou, 2010) also deduced the EOS of phonon gas in solid based on the Debye state equation (Tien and Lienhard, 1979):

$p_{\mathrm{T}}=\gamma \rho_{\mathrm{T}} C T$

where $\gamma$ is the Grüneisen constant. The density of thermomass gas is related to the medium density by(Guo, 2006; Guo, Cao et al., 2007)

$\rho_{\mathrm{T}}=\frac{\rho C T}{c^{2}}$

where $c$ is the speed of light $\left(3 \times 10^{8} \mathrm{~m} / \mathrm{s}\right)$ and $\rho$ the density of medium. It is very interesting to find that the EOS of the phonon gas in crystal is in a very similar form as that for the ideal gas (Guo, 2006) except for the proportional parameter $(\kappa$ or $\gamma$ ). Therefore we propose here a general form of EOS for ideal thermomass gas as

$p_{\mathrm{T}}=\alpha \rho_{\mathrm{T}} C T$ 
with $\alpha$ as a proportional parameter whose value is different for different state of medium. For metals, when the effect of the static electric interaction between free electrons is negligible for the thermon transport, the EOS of Eq. (6) is also available (Wang and Guo, 2010). For the thermomass gas in liquid, Eq. (6) might provide a rough approximation as well.

\subsection{Governing Equations for Transport}

Eq. (6) indicates that a higher temperature leads to a higher thermomass pressure, and that the thermomass gas will be driven to flow by a thermomass pressure difference. To derive the governing equations of the thermomass flow, we need to define the macroscopic velocity of thermomass gas flow first. Based on the thermomass theory, the mass flow rate of the thermomass gas can be expressed as

$\dot{m}_{T}=\frac{\mathbf{q}}{c^{2}}=\rho_{T} \mathbf{u}_{T}$.

Eq. (7) yields the determination of the macroscopic drift velocity as

$\mathbf{u}_{T}=\frac{\mathbf{q}}{\rho C T}$.

Eq. (8) indicates that the macroscopic velocity of thermomass gas is identical to the transport velocity of heat flow, which is calculated by the heat flux divided by the thermal energy per volume. The value of this velocity is usually very small in our normal life.

Let's consider the thermomass gas flowing by a temperature difference in a medium without any internal heat source. Suppose the thermomass gas is in local equilibrium and the governing equations for the thermomass gas transport can be derived very similarly as the classical fluid mechanics. The continuity equation is

$\frac{\partial \rho_{T}}{\partial t}+\nabla \cdot\left(\rho_{T} \mathbf{u}_{T}\right)=0$

The momentum equation is

$\rho_{T} \frac{D \mathbf{u}_{T}}{D t}+\nabla p_{T}+\mathbf{f}_{T}=0$

where $D / D t$ means the total derivative, and $\mathbf{f}_{T}$ is the resistance force per unit volume when the thermomass gas flows through the medium.

The total energy of the thermomass gas in a medium is just sum of the kinetic energy and the potential (pressure) energy, calculated by

$E_{T}=\iint_{V}\left(\rho_{T} u_{T} d u_{T}+d p_{T}\right) d V$

where $V$ is the total volume of medium,

$V=\frac{U}{c^{2} \rho_{T}}$

with $U$ representing the thermal energy in the medium.

For most engineering cases, the drift velocity of the thermomass flow is very low and the kinetic energy is negligible compared with the potential energy in Eq. (11). Furthermore, if the temperature gradient is not high, the total energy of the thermomass gas can be calculated as
$E_{T}=\alpha \frac{C}{c^{2}} U T$

which is proportional to the entransy (Guo, Zhu et al., 2007) of the medium in heat transfer.

Eq. (13) indicates that the physical essence of entransy is the (potential) energy of the thermomass. The entransy, or the potential energy of thermomass, will dissipate when heat transfers from the high to the low temperature (Guo, Zhu et al., 2007). Therefore it characterizes the irreversibility of heat transfer (Chen, Wang et al., 2009). The principle of minimizing or maximizing the entransy dispersion is also valuable to optimize the transport network micorstructure (Chen, Wang et al., 2009; Liu, Wang et al., 2010).

\section{GENERAL LAW OF HEAT CONDUCTION AND DISCUSSION}

\subsection{General Heat Conduction Equation and Its Linear Form}

Equations (9) and (10) describe the movement of the thermomass gas in media without any other artificial assumptions. Therefore, the substitutions of Eqs. (5), (6) and (8) into them yield the general heat conduction law in a continuum medium. For those with heat sources in the media, the law can be easily extended by adding a source term on the right-hand side of Eq. (9). Each of the equations and the parameters has a clear physical meaning and can be determined through measurement or analysis. The general heat conduction equation is much more complex in form compared with any other existing equations for heat conduction. Specially, for the one-dimensional case, Eq. (10) can be simplified as

$$
\frac{\partial \mathbf{q}}{\partial t}-\frac{\mathbf{q}}{T} \frac{\partial T}{\partial t}+\frac{\mathbf{q}}{\rho C T} \frac{\partial \mathbf{q}}{\partial x}-\frac{q^{2}}{\rho C T^{2}} \frac{\partial T}{\partial x}+2 \alpha \rho C^{2} T \frac{\partial T}{\partial x}+\mathbf{f}_{T} c^{2}=0
$$

The first four terms of Eq. (14) are derived from the total derivative of velocity of the thermomass gas, which reflect the thermal inertial effects. The fifth term is from the thermomass pressure driving effect, and the last term represents the resistance effect.

When the velocity of thermomass gas flow $\left(u_{T}\right)$ is very small (much lower than $1 \mathrm{~m} / \mathrm{s}$ ), the resistance is assumed to have a linear relationship with the velocity

$f_{T}=\beta u_{T}$

where $\beta$ is a parameter to be determined. Substitute Eq. (15) into Eq. (14), and the general law of heat conduction in Eq. (14) has to be consistent with the Fourier's law in Eq. (1) as long as the inertial terms are neglected. The value of $\beta$ is therefore determined as

$\beta=\frac{2 \alpha \rho^{2} C^{3} T^{2}}{c^{2} k}$

where $k$ is the thermal conductivity of the medium. This formulation of $\beta$ is also valid for $2 \mathrm{D}$ and $3 \mathrm{D}$ cases.

Therefore the general law of heat conduction in one-dimensional materials under the linear resistance assumption can be further expressed as

$\tau_{\mathrm{T}} \frac{\partial \mathbf{q}}{\partial t}-l \rho C \frac{\partial T}{\partial t}+l \frac{\partial \mathbf{q}}{\partial x}-b k \frac{\partial T}{\partial x}+k \frac{\partial T}{\partial x}+\mathbf{q}=0$ 
where

$$
\tau_{\mathrm{T}}=\frac{k}{2 \alpha \rho C^{2} T}
$$

is a characteristic time,

$$
l=\frac{q k}{2 \alpha C(\rho C T)^{2}}=u_{\mathrm{T}} \tau_{\mathrm{T}}
$$

is a characteristic length, and

$$
b=\frac{q^{2}}{2 \alpha \rho^{2} C^{3} T^{3}}
$$

is a dimensionless parameter for charactering flow compressibility of thermomass gas. For a heat flux $q=10^{4} \mathrm{~W} / \mathrm{m}^{2}$ conducting in silicon at the room temperature, $\tau_{\mathrm{T}}$ is on the order of $10^{-10} \mathrm{~s}, l$ on the order of $10^{-15}$ $\mathrm{m}$, and $b$ on the order of $10^{-15}$

\subsection{New Understanding of Fourier's Law}

Equation (1) tells empirically that the heat flux is proportional to the temperature gradient in a heat conduction process. The general law based on the thermomass gas flow model provides us a brand-new viewpoint in mechanics to understand the Fourier's law. From the determination process of $\beta$, we find that the general heat conduction law can degrade to the Fourier's law on the conditions of (i) negligible inertial effects compared with the driving force (thermomass pressure gradient or temperature gradient); and (ii) small heat flux assumption leading to the linear relationship between the resistance and the velocity of the thermomass gas. The Fourier's law essentially means the balance between the driving force and the resistant force in the thermomass fluid mechanics. Therefore the Fourier's law will break down when the inertial effect is not negligible or the heat flux is not small enough.

\subsection{Transient non-Fourier conduction}

Let's consider the unsteady non-Fourier's heat conduction first. The ultrafast heating process has many important applications and has been studied by various thermal wave models as mentioned in the Introduction. The general heat conduction law, Eq. (17), indicates that it will degenerate to the same form as the CV model if the thermal inertial effects (the second to the forth terms) are negligible, even though the physical significances of the characteristic times are different from each other. The characteristic time in the CV model may represent the relaxation time for approaching the thermodynamic equilibrium, while the one in the thermomass gas model describes the lagging response from the temperature gradient to the heat flux. Their values may be very close for metals, or distinguished significantly for pure crystals. Thanks to their quite different physical significances, the two models have different predictions for the thermal wave propagation behavior.

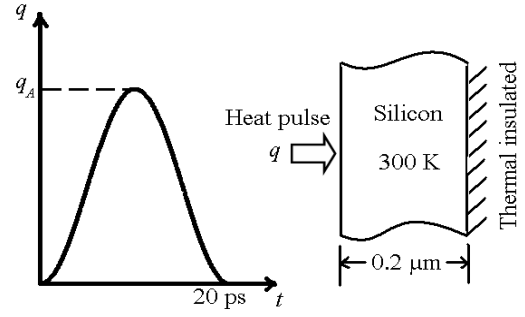

Fig. 1 Schematic of temperature response in a silicon film to one-side heat flux pulse
Let us first consider the temperature response in a silicon thin film to a heat flux pulse from the left side. The schematic diagram is shown in Fig. 1. The film is $0.2 \mu \mathrm{m}$ thick and the initial temperature is $300 \mathrm{~K}$. The heat flux pulse on the left side can be expressed as

$q(0, t)=\frac{q_{\mathrm{A}}}{2}\left(1-\cos \left(\frac{2 \pi t}{t_{0}}\right)\right)$,

when $\mathrm{t}_{0}>\mathrm{t}>0$. In our simulation, we set $q_{\mathrm{A}}=5 \times 10^{11} \mathrm{~W} / \mathrm{m}^{2}$ and $t_{0}=20 \mathrm{ps}$. The properties of the silicon film are: the density $\rho=2330 \mathrm{~kg} / \mathrm{m}^{3}$, the thermal conductivity $\mathrm{k}=163 \mathrm{~W} / \mathrm{m} \mathrm{K}$, the specific heat $\mathrm{C}=500 \mathrm{~J} / \mathrm{kg} \mathrm{K}$ and the Gruneisen constant $\gamma=1.96$. These properties lead to the characteristic time of the thermomass gas model two order of magnitudes higher than that of the CV model. Fig. 2 compares the temperature response at different time points between the two models. The thermomass gas model predicts a much slower response and a much more heterogeneous temperature distribution in the silicon film.
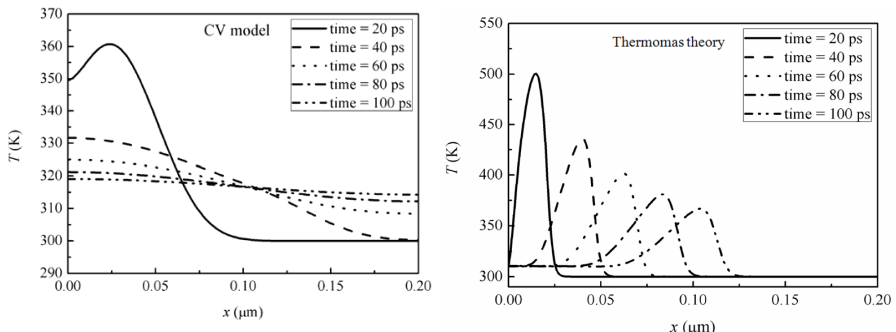

Fig. 2 Temperature distributions at different time points predicted by the two models. The parameters are $\rho=2330 \mathrm{~kg} / \mathrm{m}^{3}, \mathrm{k}=163 \mathrm{~W} / \mathrm{m}$ $\mathrm{K}, \mathrm{C}=657 \mathrm{~J} / \mathrm{kg} \mathrm{K}$ and $\gamma=1.96$.

Second, consider the thermal wave response to the two-side temperature step in a metal where the characteristic times for the two models are equal. The schematic diagram is shown in Fig. 3. The metal medium is $\mathrm{L}$ in length, and at an initial temperature, $\mathrm{T}_{0}$. When time starts $(t>0)$, the temperature at the two ends changes to $T_{w}$ and holds The equations can be consistently non-dimensionalized when $\mathrm{L}=k / \rho C \sqrt{2 C T}$. Fig. 4 compares the temperature distributions between the $\mathrm{CV}$ model and the general heat conduction law based on the thermomass theory at different time $\left(\mathrm{t}^{*}=\mathrm{t} / \tau=0.4\right.$ or 0.8$)$ for different boundary conditions $\left(\mathrm{T}_{\mathrm{w}}{ }^{*}=\mathrm{T}_{\mathrm{w}} / \mathrm{T}_{0}=0.9\right.$ or 0.3$)$. The finite difference method was used to solve both models in their non-dimensional forms, with 10000 time steps, 1000 spatial grids. Fig. 4a and b shows the results when the boundary temperature step is small $\left(\Delta \mathrm{T}^{*}=1-\mathrm{T}_{\mathrm{w}}{ }^{*}=0.1\right)$, and $\mathrm{c}$ and $\mathrm{d}$ shows the results for a high one $\left(\Delta \mathrm{T}^{*}=0.7\right)$. The results indicate that when the $\Delta T^{*}$ is small, the temperature distributions from both models are similar, except for the more wavelet characteristics from the thermomass theory making the temperature distribution more fluctuating; otherwise when the $\Delta \mathrm{T}^{*}$ is high, the results from the thermomass theory deviate significantly from the CV model. Especially, Fig. $4 \mathrm{~d}$ shows that when the two waves from the boundary temperature step meet, the $\mathrm{CV}$ model leads to an unphysical temperature distribution under zero, similar as reported in Ref. (Bai and Lavine, 1995; Korner and Bergmann, 1998), while the thermomass theory will result in a reasonable temperature distribution with considering the thermal inertial effects.

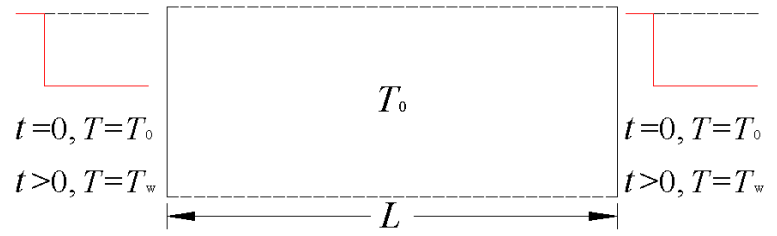

Fig. 3 Schematic of temperature response to two-side temperature step 

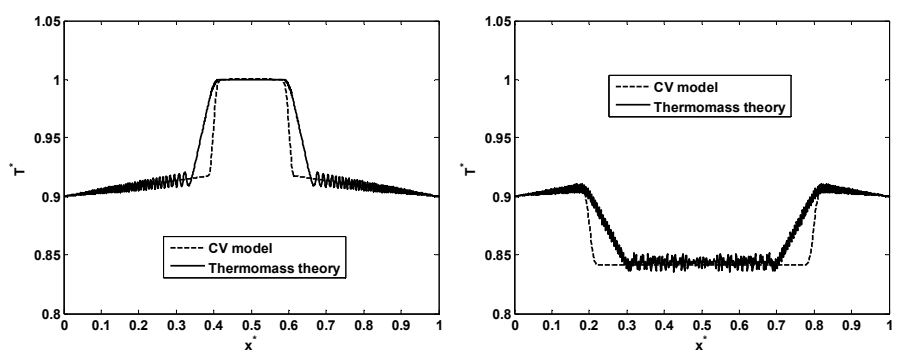

(a) $\mathrm{T}_{\mathrm{w}}{ }^{*}=0.9, \mathrm{t}^{*}=0.4$

(b) $\mathrm{T}_{\mathrm{w}}^{*}=0.9, \mathrm{t}^{*}=0.8$;
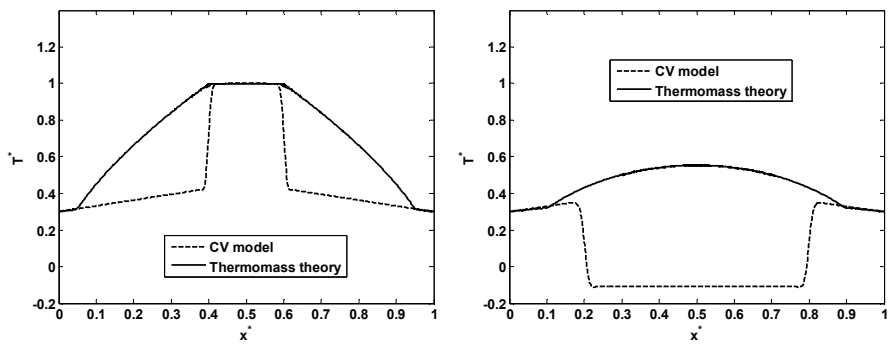

(c) $\mathrm{T}_{\mathrm{w}}{ }^{*}=0.3, \mathrm{t}^{*}=0.4$;

(d) $\mathrm{T}_{\mathrm{w}}{ }^{*}=0.3, \mathrm{t}^{*}=0.8$;

Fig. 4 Thermal wave propagation behavior for different boundary conditions. The non-dimensional parameters are defined as $\mathrm{x}^{*}=\mathrm{x} / \mathrm{L}, \mathrm{T}^{*}=\mathrm{T} / \mathrm{T}_{0}$, and $\mathrm{t}^{*}=\mathrm{t} / \tau$.

\subsection{High-rate steady non-Fourier conduction}

Generally the non-Fourier conduction is used to being referred to the ultrafast/transient heat conduction phenomena, which it is actually beyond. The general heat conduction equation, Eq. (14), indicates that the thermal inertial effect may be non-negligible for high-rate heating even at the steady state. Such an effect has never been well studied because of lack of corresponding theories.

The thermomass gas model gives the heat conduction governing equations in one-dimensional materials by

$\frac{\partial \mathbf{q}}{\partial x}=0$

$\frac{\mathbf{q}}{\rho C T} \frac{\partial \mathbf{q}}{\partial x}-\frac{q^{2}}{\rho C T^{2}} \frac{\partial T}{\partial x}+2 \alpha \rho C^{2} T \frac{\partial T}{\partial x}+\frac{2 \alpha \rho C^{2} T}{k} \mathbf{q}=0$,

which lead to

$\left(1-\frac{q^{2}}{2 \alpha \rho^{2} C^{3} T^{3}}\right) k \frac{\partial T}{\partial x}+\mathbf{q}=0$

Eq. (24) governs the high-rate steady non-Fourier heat conduction, with $\mathrm{k}$ representing the intrinsic thermal conductivity. The equation indicates that the effective thermal conductivity is lower than the intrinsic one when the thermal inertial effect is considered. When the heat flux is low, Eq. (24) will degenerate to the classical Fourier's law for the steady-state heat conduction. Usually the effective thermal conductivity measured by experiments are defined as

$k_{e f f}=\frac{q L}{\Delta T}$

where $\mathrm{L}$ is the length of the material and $\Delta \mathrm{T}$ the temperature difference.
Fig. 5 shows the effective thermal conductivity varying with the length and the temperature of silicon nanowires for a given temperature difference $(\Delta \mathrm{T}=100 \mathrm{~K})$. The material properties used in the predictions are the same as those in Fig. 2. The average temperature is $110 \mathrm{~K}$ for Fig. 5a, and the nanowire length is $100 \mathrm{~nm}$ for Fig. 5b. The result in Fig. 5a indicate that the effective thermal conductivity of silicon nanowires decreases with the decreasing length for given temperatures of both ends. This result agrees qualitatively with other theoretical or numerical results (Fujii, Zhang et al., 2005; Mingo and Broido, 2005; Donadio and Galli, 2009). A shorter nanowire results in higher temperature gradient and higher thermal inertial effect, and therefore a lower effective thermal conductivity as a result. When the material is long enough, such longer than $10 \mu \mathrm{m}$ for this case, the effective thermal conductivity is identical to the bulk one, which means the thermal inertial effect is negligible. Fig. 5b shows the effective thermal conductivity increases with the average temperature of the nanowire for give $\Delta \mathrm{T}$ and $\mathrm{L}$. This result agree qualitatively well with the experimental data in Ref. (Fujii, Zhang et al., 2005; Chen, Hochbaum et al., 2008; Hochbaum, Chen et al., 2008).

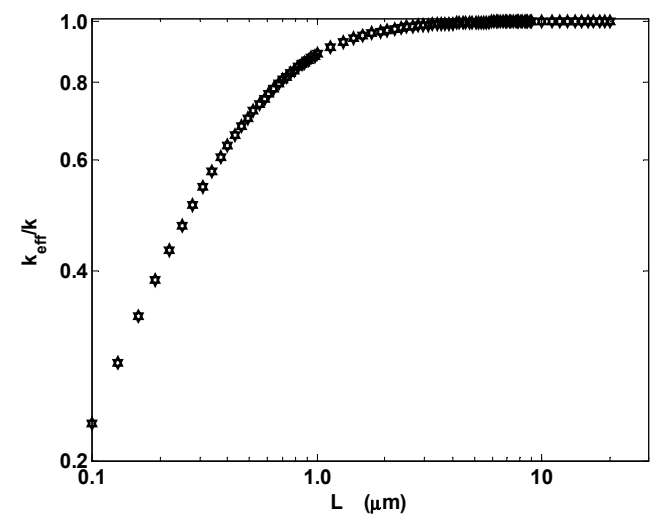

(a) Effective thermal conductivity vs. length of nanowire

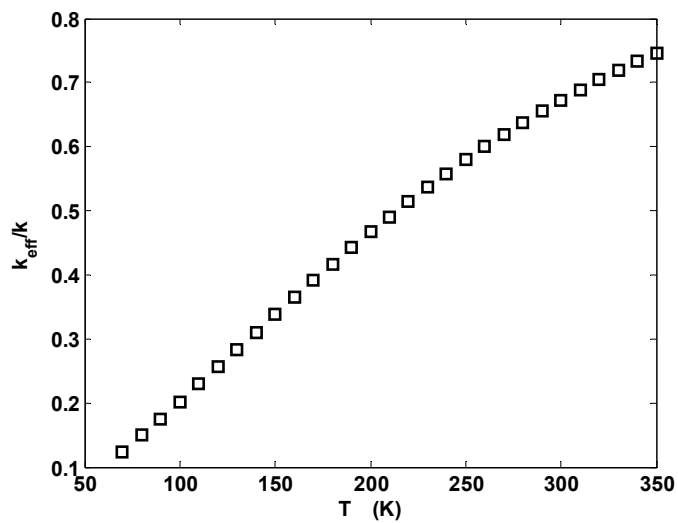

(b) Effective thermal conductivity vs. average temperature

Fig. 5 The effective thermal conductivity changing with the length and the average temperature of silicon nanowaires for a given temperature difference $(\Delta \mathrm{T}=100 \mathrm{~K})$. The parameters used are $\rho=2330 \mathrm{~kg} / \mathrm{m}^{3}, \mathrm{k}=163 \mathrm{~W} / \mathrm{m} \mathrm{K}, \mathrm{C}=500 \mathrm{~J} / \mathrm{kg} \mathrm{K}$ and $\gamma=1.96$.

\section{CONCLUSIONS}

(1) We have built up a thermomass gas model based on the thermomass theory, which regards heat owning mass-energy duality, exhibiting energy-like features in conversion and mass-like features in transfer processes. The equivalent mass of thermal energy is determined based on the mass-energy equivalence of Einstein, which therefore leads to 
the inertia of heat in transfer. The thermomass gas model describes the heat conduction as fluid-flow-like process in a medium, and derives the equation of state and the governing equations for transport based on methodologies of the classical mechanics since the drift speed of the thermomass gas is far lower than the speed of light. We therefore present the general heat conduction law to describe the relationship between the heat flux and the temperature field.

(2) The general law degenerates to the Fourier's law if all the thermal inertial effects are negligible, and therefore provides us a new viewpoint to understand the Fourier's law for heat conduction. The Fourier's law essentially means the balance between the driving force and the resistant force in the thermomass fluid mechanics. Therefore the Fourier's law will break down when the inertial effect is not negligible or the linear resistance-velocity relationship is not valid.

(3) The general law degenerates to the same form as the CV model with different physical significances for the characteristic time, when the time dependent parts of the thermal inertial effects are only considered. The characteristic time in the thermomass gas model means the lagging time from the temperature gradient to the corresponding heat flux, while the one in the $\mathrm{CV}$ model is the relaxation time from the thermal non-equilibrium to the equilibrium state. This difference leads to very different predictions for the thermal wave propagation behavior. For the dielectrics, the values of the characteristic time may differ by two orders of magnitude, which results in a much slower temperature response to a heat pulse predicted by the thermomass gas model than that by the CV model. Even for the heat waves in metals, where the two characteristic times are very close, the thermal inertial effects also cause different features of heat wave propagation. Especially the unphysical temperature distribution under zero predicted by the $\mathrm{CV}$ model, when two low-temperature cooling waves meet, will not appear in the predictions by our general law.

(4) The general law describes the steady-state non-Fourier heat conduction when the time dependent terms are neglected, with the thermal inertial effects considered. The predictions show that the effective thermal conductivity of silicon nanowires, which is smaller than the intrinsic one, decreases with the decreasing length for a given temperature difference between ends, and increases with the average temperature for a given length. These results agree qualitatively with the existing theoretical or experimental data.

\section{ACKNOWLEDGEMENTS}

This work is supported by the National Natural Science Foundation of China (Grant No. 50606018, 50730006 and 50976053) and the LANL's LDRD Project 20080727PRD2 through the J. R. Oppenheimer Fellowship awarded to M.W. The authors would like to thank helpful discussions with Dr. Hongye Zhu, Dr. Qingguang Zhang, Dr. Quanwen Hou, Dr. Haidong Wang, and Mr. Yinshan Wang.

\section{NOMENCLATURE}

$b \quad$ Dimensionless parameter for thermal inertia

$c \quad$ Light speed

C Specific heat

$f \quad$ Resistance

$k \quad$ Thermal conductivity

$l \quad$ Characteristic length

$u \quad$ Velocity

$V \quad$ Volume

$p \quad$ Pressure

$q \quad$ Heat flux

$u \quad$ Velocity

$U \quad$ Thermal energy

$t \quad$ Time

$T \quad$ Temperature

$x, y \quad$ Coordinates

$\begin{array}{ll}\text { Greek symbols } \\ \alpha & \text { Parameter for EOS of thermomass gas } \\ \beta & \text { Proportionality coefficient for resistance } \\ \gamma & \text { Grüneisen constant } \\ \kappa & \text { Ratio of specific heats } \\ \xi & \text { Effects of interaction between thermons } \\ \rho & \text { Density } \\ \tau & \text { Characteristic time }\end{array}$

$\begin{array}{ll}\text { Subscripts } \\ 0 & \text { Initial } \\ \mathrm{CV} & \text { CV model } \\ \text { eff } & \text { Effective } \\ \mathrm{T} & \text { Thermomass gas } \\ \mathrm{w} & \text { Wall }\end{array}$

\section{REFERENCES}

Ali, A.H., 1999, "Statistical mechanical derivation of Cattaneo's heat flux law," Journal of Thermophysics and Heat Transfer 13(4), 544-546. doi: $10.2514 / 2.6474$

Ali, Y.M. and Zhang, L.C., 2005, "Relativistic heat conduction," International Journal of Heat and Mass Transfer 48, 2397-2406. doi: 10.1016/j.ijheatmasstransfer.2005.02.003

Bai, C. and Lavine, A.S., 1995, "On hyperbolic heat-conduction and the 2nd law of thermodynamics," Journal of Heat Transfer-Transactions of the ASME 117(2), 256-263.

doi: $10.1115 / 1.2822514$

Barreiro, A., Rurali, R., et al., 2008, "Subnanometer motion of cargoes driven by thermal gradients along carbon nanotubes," Science 320(5877), 775-778.

doi: $10.1126 /$ science. 1155559

Brorson, S.D., Fujimoto, J.G., et al., 1987, "Femtosecond electronic heat-transport dynamics in thin gold films," Physical Review Letters 59(17), 1962-1965.

doi: 10.1103/PhysRevLett.59.1962

Cattaneo, C., 1958, "Sur Une Forme De Lequation De La Chaleur Eliminant Le Paradoxe Dune Propagation Instantanee," Comptes Rendus Hebdomadaires Des Seances De L Academie Des Sciences 247(4), 431-433.

Chen, Q., Wang, M.R., et al., 2009, "Irreversibility of Heat Conduction in Complex Multiphase Systems and Its Application to the Effective Thermal Conductivity of Porous Media," International Journal of Nonlinear Sciences and Numerical Simulation 10(1), 57-66.

Chen, Q., Wang, M.R., et al., 2009, "Optimization Principle for Variable Viscosity Fluid Flow and Its Application to Heavy Oil Flow Drag Reduction," Energy \& Fuels 23, 4470-4478.

doi: $10.1021 / \mathrm{ef} 900107 \mathrm{~b}$

Chen, R., Hochbaum, A.I., et al., 2008, "Thermal conductance of thin silicon nanowires," Physical Review Letters 101(10), 105501. doi: 10.1103/PhysRevLett.101.105501

Chester, M., 1963, "Second Sound in Solids," Physical Review 131(5), 2013-2015.

doi: 10.1103/PhysRev.131.2013

Coleman, B.D., Fabrizio, M., et al., 1982, "On the thermodynamics of 2nd sound in dieletric crystals," Archive for Rational Mechanics and Analysis 80(2), 135-158.

doi: $10.1007 / \mathrm{BF} 00250739$ 
Donadio, D. and Galli, G., 2009, "Atomistic Simulations of Heat Transport in Silicon Nanowires," Physical Review Letters 102(19), 195901.

doi: 10.1103/PhysRevLett.102.195901

Eckart, C., 1940, "The thermodynamics of irreversible processes III Relativistic theory of the simple fluid," Physical Review 58(10), 919924.

doi: 10.1103/PhysRev.58.919

Einstein, A., Lorentz, H.A., et al., 1952, The principle of Relativity, Dover Publications, New York.

Fourier, J., 1955, Analytical Theory of Heat, Dover Publications, New York.

Fujii, M., Zhang, X., et al., 2005, "Measuring the thermal conductivity of a single carbon nanotube," Physical Review Letters 95(6), 065502. doi: 10.1103/PhysRevLett.95.065502

Guo, Z.Y., 2006, "Motion and transfer of thermal mass-Thermal mass and thermon gas," Journal of Engineering Thermophysics 27(4), 631634.

Guo, Z.Y., Cao, B.Y., et al., 2007, "State equation of phonon gas and conservation equations for phonon gas motion," Acta Physica Sinica 56(6), 3306-3312

Guo, Z.Y. and Hou, Q.W., 2010, "Thermal Wave Based on the Thermomass Model," Journal of Heat Transfer-Transactions of the ASME 132(7), 072403.

doi: $10.1115 / 1.4000987$

Guo, Z.Y. and Zhu, H.Y., 2007, "Motion and transfer of thermal massconservation equations of thermon gas and Fourier's law," Journal of Engineering Thermophysics 28(1), 86-88.

Guo, Z.Y., Zhu, H.Y., et al., 2007, "Entransy - A physical quantity describing heat transfer ability," International Journal of Heat and Mass Transfer 50(13-14), 2545-2556.

doi: 10.1016/j.ijheatmasstransfer.2006.11.034

Gurtin, M.E. and Pipkin, A.C., 1968, "A general theory of heat conduction with finite wave speeds," Archive for Rational Mechanics and Analysis 31(2), 113-126.

doi: 10.1007/BF00281373

Guyer, R.A. and Krumhans.Ja, 1966, "Solution of linearized phonon Boltzmann equation," Physical Review 148(2), 766-778.

doi: 10.1103/PhysRev.148.766

Hochbaum, A.I., Chen, R.K., et al., 2008, "Enhanced thermoelectric performance of rough silicon nanowires," Nature 451(7175), 163-U165. doi: 10.1038 /nature06381

Joseph, D.D. and Preziosi, L., 1989, "Heat waves," Reviews of Modern Physics 61(1), 41-73.

doi: 10.1103/RevModPhys.61.41

Korner, C. and Bergmann, H.W., 1998, "The physical defects of the hyperbolic heat conduction equation," Applied Physics a-Materials Science \& Processing 67(4), 397-401.

doi: $10.1007 / \mathrm{s} 003390050792$

Landau, L.D. and Lifshitz, E.M., 1959, Fluid Mechanics, Pergamon Press, London.

Liu, X.B., Wang, M.R., et al., 2010, "Minimum entransy dissipation principle for the optimization of transport networks," International Journal of Nonlinear Sciences and Numerical Simulation In Press.

Maurer, M.J., 1969, "Relaxation Model for Heat Conduction in Metals," Journal of Applied Physics 40(13), 5123-5130. doi: $10.1063 / 1.1657362$

Mendoza, E., 1961, "A sketch for a history of early thermodynamics," Physics Today 14(2), 32-42.

doi: $10.1063 / 1.3057388$

Mingo, N. and Broido, D.A., 2005, "Length dependence of carbon nanotube thermal conductivity and the "problem of long waves"," Nano Letters 5(7), 1221-1225.

doi: $10.1021 / \mathrm{n} 1050714 \mathrm{~d}$

Nernst, W., 1918, Die Theoretischen Grundlagen Des Neuen Wärmesatzes.

Onsager, L., 1931, "Reciprocal relations in irreversible processes. I," Physical Review 37(4), 405-426.

doi: 10.1103/PhysRev.37.405

Qiu, T.Q. and Tien, C.L., 1993, "Heat transfer mechanisms during short-pulse laser heating of metals," Journal of Heat TransferTransactions of the Asme 115(4), 835-841. doi: $10.1115 / 1.2911377$

Qiu, T.Q. and Tien, C.L., 1994, "Femtosecond laser heating of multilayer metals-I. Analysis," International Journal of Heat and Mass Transfer 37(17), 2789-2797.

doi: $10.1016 / 0017-9310(94) 90396-4$

Rubin, M.B., 1992, "Hyperbolic heat conduction and the second law," International Journal of Engineering Science 30(11), 1665-1676. doi: 10.1016/0020-7225(92)90134-3

Tien, C.L. and Lienhard, J.H., 1979, Statistical Thermodynamics, Hemisphere Publishing Corporation, Washington D.C.

Tolman, R.C., 1930, "On the weight of heat and thermal equilibrium in general relativity," Physical Review 35(8), 0904-0924.

doi: 10.1103/PhysRev.35.904

Tzou, D.Y., 1989, "Shock wave formation around a moving heat source in a solid with finite speed of heat propagation," International Journal of Heat and Mass Transfer 32(10), 1979-1987. doi: 10.1016/0017-9310(889)990166-X

Tzou, D.Y., 1991, "The resonance phenomenon in thermal waves," International Journal of Engineering Science 29(9), 1167-1177. doi: 10.1016/0020-7225(91)90119-N

Tzou, D.Y., 1995, "Experimental support for the lagging behavior in heat propagation," Journal of Thermophysics and Heat Transfer 9(4), 686-693.

doi: $10.2514 / 3.725$

Tzou, D.Y., 1995, "The generalized lagging response in small-scale and high-rate heating " International Journal of Heat and Mass Transfer 38(17), 3231-3240.

doi: 10.1016/0017-9310(95)00052-B

Tzou, D.Y. and Chiu, K.S., 2001, "Temperature-dependent thermal lagging in ultrafast laser heating," International Journal of Heat and Mass Transfer 44(9), 1725-1734.

doi: 10.1016/S0017-9310(00)00215-5

Tzou, D.Y. and Zhang, Y.S., 1995, "An analytical study on the fasttransient process in small scales," International Journal of Engineering Science 33(10), 1449-1463. doi: 10.1016/0020-7225(94)00130-C

Vernotte, P., 1958, "Les Paradoex De La Theorie Continue De Lequation De La Chaleur," Comptes Rendus Hebdomadaires Des Seances De L Academie Des Sciences 246(22), 3154-3155. 
Vining, C.B., 2008, "Materials science - Desperately seeking silicon," Nature 451(7175), 132-133.

doi: $10.1038 / 451132 \mathrm{a}$

Wang, H.D. and Guo, Z.Y., 2010, "Thermon gas - the thermal energy carrier in gas and metal," Chinese Science Bullitin In Press.
$\mathrm{Xu}$, M.T. and Wang, L.Q., 2002, "Thermal oscillation and resonance in dual-phase-lagging heat conduction," International Journal of Heat and Mass Transfer 45(5), 1055-1061. doi: 10.1016/S0017-9310(01)00199-5

Zhang, Q.G., Cao, B.Y., et al., 2006, "Motion and transfer of thermal mass-Equation of state for thermon gas," Journal of Engineering Thermophysics 27(6), 908-910. 